\title{
Genomic structure and expression of the human serotonin $2 A$ receptor gene (HTR2A) locus: identification of novel HTR2A and antisense (HTR2A-AS1) exons
}

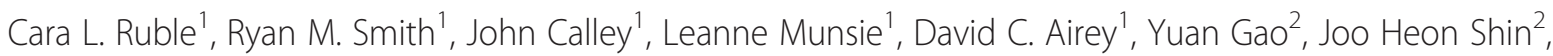
Thomas M. Hyde ${ }^{2,3}$, Richard E. Straub2, Daniel R. Weinberger ${ }^{2,4}$ and Laura K. Nisenbaum ${ }^{1 *}$

\begin{abstract}
Background: The serotonin 2A receptor is widely implicated in genetic association studies and remains an important drug target for psychiatric, neurological, and cardiovascular conditions. RNA sequencing redefined the architecture of the serotonin 2A receptor gene (HTR2A), revealing novel mRNA transcript isoforms utilizing unannotated untranslated regions of the gene. Expression of these untranslated regions is modulated by common single nucleotide polymorphisms (SNPs), namely rs6311. Previous studies did not fully capture the complexity of the sense- and antisense-encoded transcripts with respect to novel exons in the HTR2A gene locus. Here, we comprehensively catalogued exons and RNA isoforms for both HTR2A and HTR2A-AS1 using RNA-Seq from human prefrontal cortex and multiple mouse tissues. We subsequently tested associations between expression of newfound gene features and common SNPs in humans.
\end{abstract}

Results: We find that the human HTR2A gene spans $\sim 66$ kilobases and consists of 7, rather than 4 exons. Furthermore, the revised human HTR2A-AS1 gene spans $\sim 474$ kilobases and consists of 18, rather than 3 exons. Three HTR2A exons directly overlap with HTR2A-AS1 exons, suggesting potential for complementary nucleotide interactions. The repertoire of possible mouse Htr2a splice isoforms is remarkably similar to humans and we also find evidence for overlapping sense-antisense transcripts in the same relative positions as the human transcripts. rs6311 and SNPs in high linkage disequilibrium are associated with HTR2A-AS1 expression, in addition to previously described associations with expression of the extended $5^{\prime}$ untranslated region of HTR2A.

Conclusions: Our proposed HTR2A and HTR2A-AS1 gene structures dramatically differ from current annotations, now including overlapping exons on the sense and anti-sense strands. We also find orthologous transcript isoforms expressed in mice, providing opportunities to elucidate the biological roles of the human isoforms using a model system. Associations between rs6311 and expression of HTR2A and HTR2A-AS1 suggest this polymorphism is capable of modulating the expression of the sense or antisense transcripts. Still unclear is whether these SNPs act directly on the expression of the sense or antisense transcripts and whether overlapping exons are capable of interacting through complimentary base-pairing. Additional studies are necessary to determine the extent and nature of interactions between the SNPs and the transcripts prior to interpreting these findings in the context of phenotypes associated with HTR2A.

Keywords: Serotonin, HTR2A, Alternative splicing, Antisense RNA, mRNA expression, Regulatory polymorphism, Allelic expression, Comparative genomics, Long non-coding RNA, 5-HT2A

\footnotetext{
*Correspondence: nisenbaum_laura_k@lilly.com

${ }^{1}$ Tailored Therapeutics, Lilly Corporate Center, Eli Lilly and Company,

Indianapolis, IN,

Full list of author information is available at the end of the article
}

\section{) Biomed Central}

C 2016 Ruble et al. Open Access This article is distributed under the terms of the Creative Commons Attribution 4.0 International License (http://creativecommons.org/licenses/by/4.0/), which permits unrestricted use, distribution, and reproduction in any medium, provided you give appropriate credit to the original author(s) and the source, provide a link to the Creative Commons license, and indicate if changes were made. The Creative Commons Public Domain Dedication waiver (http://creativecommons.org/publicdomain/zero/1.0/) applies to the data made available in this article, unless otherwise stated. 


\section{Background}

The serotonin $2 \mathrm{~A}$ receptor $\left(5-\mathrm{HT}_{2 \mathrm{~A}}\right)$ is a $\mathrm{G}$ proteincoupled receptor (GPCR) that serves as a primary target for serotonin signaling and is expressed on many cell types in the brain and periphery. $5-\mathrm{HT}_{2 \mathrm{~A}}$ remains of great scientific interest due to its many roles in normal biological functions, which include cerebral cortex excitability [1], platelet aggregation [2], smooth muscle contraction [3], vasoconstriction and dilation [4], inflammatory processes [5], and hormone signaling [6]. Among the many tissues expressing $5-\mathrm{HT}_{2 \mathrm{~A}}$, it is especially prevalent in the cerebral cortex, enriched at the apical dendrites of pyramidal neurons $[7,8]$. Commensurate with its broad biological influence, studies examining single nucleotide polymorphisms (SNPs) in the gene encoding $5-\mathrm{HT}_{2 \mathrm{~A}}$ (HTR2A) have identified more than 100 genotypic associations with a wide range of phenotypes, especially brain-related disorders [9]. Drugs that directly or indirectly modulate serotonergic signaling through $5-\mathrm{HT}_{2 \mathrm{~A}}$ receptors are used to treat neuropsychiatric, neurologic, and cardiovascular conditions, and $5-\mathrm{HT}_{2 \mathrm{~A}}$ is an emerging drug target for a variety of other indications [10-13].

Transcriptome profiling in human tissues has greatly increased our appreciation for RNA isoform diversity, revealing pervasive alternative splicing and transcription start and termination sites for nearly all multiexon genes $[14,15]$. Consequently, we can utilize this information to better understand disease states and tailor therapeutics [16], but this requires in-depth characterization of RNA expression for important targets, such as HTR2A. Knowledge of the different exons and mRNAs for HTR2A has evolved over the past 20 years. Human HTR2A complementary DNA (cDNA) was first cloned in 1991 (GenBank X57830.1) [17], followed in 1992 by a genomic structure consisting of 3 exons spanning 20kB [18]. The original Reference Sequence (RefSeq) mRNA annotation (NM_000621.1) reflects these findings. Since this first annotation, HTR2A has undergone three revisions, which removed cloning sites and altered alleles at polymorphic sites (NM_000621.2), added a 5' exon (NM_000621.3), and extended the 3' untranslated region (UTR), adding a consensus polyadenylation signal (NM_000621.4). The current gene model consists of 4 exons, spanning more than $65 \mathrm{kB}$, and also includes evidence for alternative splicing of exon 2 (NM_001165947). Based on length, these two RefSeq annotations (NM_000621.4 and NM_001165947.2) likely reflect the transcripts detected by Northern blot in 1990 [19]. There are additional HTR2A splice variants described in the literature that are not yet annotated in RefSeq. This includes an RNA isoform containing a novel 118-bp exon residing in intron 3 of the current annotation [20] and four additional exon boundary changes resulting in novel RNA isoforms: a truncated exon 2, a retained intron between exons 1 and 2, a 5' extension of exon 1, and a 3' extension of exon 4 [21].

The HTR2A locus also hosts a long non-coding RNA (lncRNA) gene on the antisense strand, annotated as HTR2A-AS1 in RefSeq, and encoding two alternatively spliced isoforms (NR_103752 and NR_046612). Four Expressed Sequence Tags (ESTs) (AI076014.1, AI216351.1, AI914390.1, and AW469493.1) suggest the existence of at least three different splice variants, some of which are detectable following PCR amplification of cDNA synthesized from human brain and testes [21, 22]. Furthermore, HTR2A-AS1 expression is readily detectable in human testes according to RNA-Seq data from the Genotype-Tissue Expression (GTEx) project [23]. However, expression is sparse in other GTEx-investigated tissues, including the cerebral cortex (www.GTExportal.org).

The $5-\mathrm{HT}_{2 \mathrm{~A}}$ gene in mice spans approximately $66 \mathrm{kB}$ on chromosome 14 and contains 3 exons. The mouse gene shares many similarities with the human gene. For example, the relative genomic spacing of the protein-coding exons and the use of constitutive splice sites is conserved, such that the mouse exon 1/human exon 2 both encode the first 138 amino acids of 5-HT2A, followed by a relatively short intron $(2.6 \mathrm{kB}$ in mouse, $2.9 \mathrm{kB}$ in human). Mouse exon 2/human exon 3 both encode the subsequent 67 amino acids, followed by a relatively large intron (60.4kB in mouse, $56.8 \mathrm{kB}$ in humans). Mouse exon $3 / \mathrm{hu}$ man exon 4 both encode the remaining 266 amino acids. However, mouse Htr $2 a$ currently lacks in-depth analysis of RNA isoform expression to delineate alternative transcripts, as previously performed on human HTR2A. Therefore, it is unclear how well the mouse recapitulates the molecular biology of human HTR2A splice variants, limiting its capacity as a model organism for understanding HTR2A expression.

At the crux of the many genotype-phenotype associations involving HTR2A is whether the implicated genetic variants have functional consequences. The most frequently cited SNPs in studies concerning HTR2A are rs6311 and rs6313. rs6311 and rs6313 are in near-perfect linkage disequilibrium (LD) [24], located 1538 bases apart on chromosome 13, and neither alter the encoded protein. rs6311 (also known as -1438G > A and A-1438G) is historically described as being located in the upstream or promoter region of HTR2A. However, rs6311 is transcribed in minor isoforms of HTR2A mRNA expressed in the brain, perhaps through the use of an alternative transcription start site that results in an extended 5' UTR [21]. The variant "A" allele of rs6311 is associated with reduced expression of isoforms containing this 5 ' UTR extension. rs6311 has also been correlated with total HTR2A mRNA and protein expression $[25,26]$, although multiple studies failed to find an allele-specific effect of rs6311 on the most abundant primary transcript isoform [21, 27, 28]. rs6313 
(also known as T102C) is a synonymous SNP residing in exon two. Because rs6313 lacks strong functional evidence and is in high LD with rs6311, genetic associations with rs6313 are often attributed to the functional consequences of rs6311.

Understanding the functional consequences of SNPs is a critical first step towards appreciating their roles in disease. To date, only a single study has examined HTR $2 A$ SNPs and the expression of the extended 5' UTR isoform in affected tissues, finding no difference between postmortem brain tissue from autistic individuals and controls [29]. To better understand the role of HTR2A in human disease, here we have comprehensively catalogued the transcripts expressed from the HTR2A gene locus in the dorsolateral prefrontal cortex (DLPFC) from unaffected controls and schizophrenia patients. We also characterized Htr $2 a$ locus expression in the mouse, contrasting it with our human findings to determine where mice could provide models for studying human $5-\mathrm{HT}_{2 \mathrm{~A}}$ function. Following, we asked whether rs6311 and rs6313 are associated with sense or antisense transcript expression.

\section{Results}

\section{HTR2A exons defined by splice junction analysis}

In addition to the four annotated exons, we found evidence for four different alternative exons, including two that have not been previously described (exons 0 and $3 \mathrm{~b}$; Table 1). The revised gene model is presented in Fig. $1 \mathrm{~b}$. The majority of exon-exon junction reads join exons from previously annotated isoforms. However, novel exons also share splice junctions with the previously annotated exons, suggesting that they are included in mature RNA transcripts (Table 2; Additional file 1: Figure S1). The strength of predicted splice donor/acceptor sites and branch point sequences, assessed in silico using the Human Splicing Finder (http://www.umd.be/HSF/) [30], do not significantly differ across the novel and annotated exons (Additional file 2: Figure S2a). Furthermore, the stretch of nucleotides 5 to 40 bases upstream of the novel exons, presumably the polypyrimidine tracts, are more highly enriched for pyrimidine and uracil nucleotides relative to the annotated exons (Additional file 2:Figure S2a). We also used the Alternative Splice Site Predictor (http://wangcomputing.com/assp/index.html) [31] to identify and score any possible splice donor and acceptor site across the entire HTR2A locus. We subsequently compared scores at annotated and novel exons versus all other predicted donor and acceptor sites in HTR2A. Again, we found that average scores for the novel exons did not significantly differ from the annotated exons, which both scored higher than all other predicted donor/acceptor sites (Additional file 2: Figure S2b).

Exons 1, 1int, 1ext, and 0 do not have exon-exon junctions defining their 5' boundaries and are therefore considered as transcription initiation sites. The parsimonious interpretation based on RNA-Seq coverage in this area supports the existence of two transcription start sites. Transcripts containing exons 0,1 ext, and at least some of lint likely use a transcription initiation site upstream of the annotated exon 1, consistent with previous reports [21]. However, the majority of transcripts likely utilize the transcription initiation site beginning with exon 1, given the large increase in read depth at this exon (Additional file 3: Figure S3). Exons $3 \mathrm{~b}$ and 4 do not have exon-exon junctions defining their 3 ' boundaries. Exon 4 contains a canonical poly-adenylation (poly-A) signal (AATAAA) at position chr13:47405698-47405703, after which the number of mapped reads drops off precipitously, signaling the termination of the major transcript isoform(s) (Additional file 4: Figure S4). Consistent with previous findings, we observed a continuation of the 3' UTR that extends beyond the canonical poly-A signal [21], continuing $5.6 \mathrm{kB}$ distally from the annotated 3' UTR to a cluster of four canonical poly-A signals within a stretch of 88 nucleotides

Table 1 HTR2A Exons

\begin{tabular}{llll}
\hline Exon & Size $(\mathrm{bp})$ & Coordinates $^{\mathrm{a}}$ & Notes \\
\hline 0 & 187 & chr13:47472087-47472273 & Newly described \\
1 ext & 1465 & chr13:47470809-47472273 & Described in Smith et al., 2013 \\
$1 \mathrm{int}$ & 2644 & chr13:47469630-47472273 & Described in Smith et al., 2013 \\
1 & 403 & chr13:47470809-47471211 & As annotated in RefSeq \\
2 & 740 & chr13:47469630-47469825 & As annotated in RefSeq \\
$2 \mathrm{tr}$ & 196 & chr13:47469630-47470369 & Described in Smith et al., 2013 \\
3 & 201 & chr13:47466525-47466725 & As annotated in RefSeq \\
$3 a$ & 118 & chr13:47458674-47458791 & Described in Guest et al., 2000 \\
$3 b$ & 1175 & chr13:47456022-47457197 & Newly described \\
4 & 4098 & chr13:47405677-47409774 & As annotated in RefSeq \\
4 ext & 9716 & chr13:47400058-47409774 & Partially described in Smith et al., 2013 \\
\hline
\end{tabular}

${ }^{a}$ Coordinates reflect hg19/GRCh37 


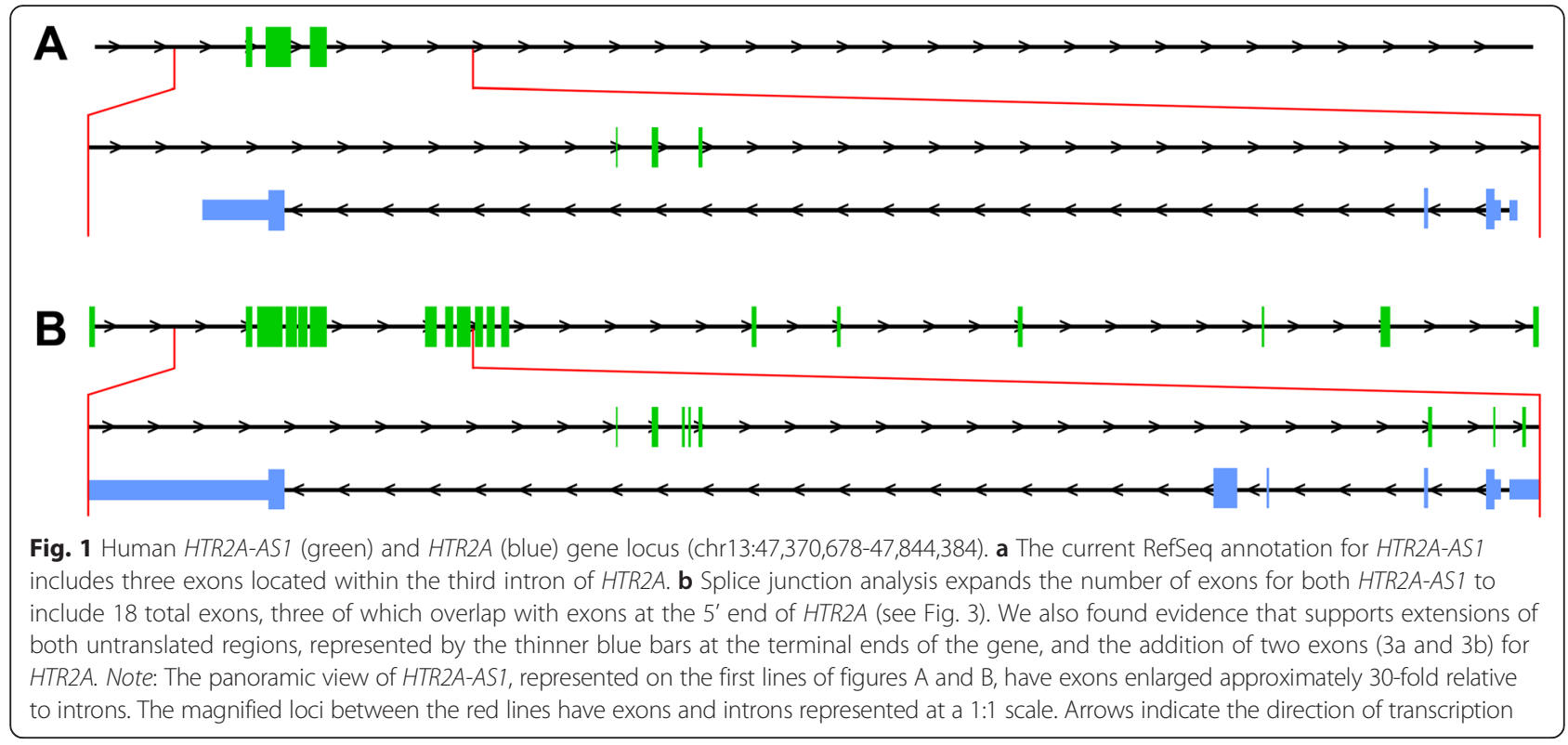

(chr13:47400058-47400144; Additional file 4: Figure S4). The lack of an exon-exon junction read to define the 3' boundary of exon $3 \mathrm{~b}$ suggests that it is unlikely to be spliced into transcripts containing exon 4 and could represent a terminal exon. Reduced read coverage following a cluster of canonical and non-canonical poly-A signals supports the interpretation that exon $3 \mathrm{~b}$ is a terminal exon (Additional file 5: Figure S5).

\section{Transcript assembly for HTR2A}

We assembled likely mRNA isoforms, excluding junctions where we see only single junction counts. We then

Table 2 HTR2A Splice Junctions

\begin{tabular}{llll}
\hline Splices From: & Splices To: & Total Junction Counts & Samples Observed \\
\hline 0 & 2 & 28 & 21 \\
0 & 2 tr & 1 & 1 \\
0 & 3 & 126 & 73 \\
0 & 4 & 1 & 1 \\
1 or 1ext & 2 & 15802 & 205 \\
1 or 1ext & 2 tr & 45 & 39 \\
1 or 1ext & 3 & 16 & 14 \\
1 or 1ext & $3 b$ & 1 & 1 \\
2 or 1int & 3 & 15253 & 200 \\
2 or 1int & $3 b$ & 1 & 1 \\
3 & $3 a$ & 72 & 54 \\
3 & $3 b$ & 39 & 34 \\
3 & 4 or 4 ext & 14221 & 203 \\
$3 a$ & $3 b$ & 26 & 19 \\
$3 a$ & 4 or 4 ext & 108 & 60 \\
\hline
\end{tabular}

\footnotetext{
${ }^{\mathrm{a} S u m m e d}$ across all samples
}

assessed the predicted open reading frames and calculated the number of predicted transmembrane (TM) domains (Table 3). Only transcript isoforms utilizing exons 2,3 , and 4 appear capable of translating protein isoforms with all 7TM domains quintessential of GPCRs.

\section{HTR2A-AS1 exons defined by splice junction analysis}

HTR2A-AS1 is on the forward strand of chromosome 13. Definition of HTR2A-AS1 splice junctions followed the same rules regarding canonical splicing as applied to $H T R 2 A$, allowing for stranded assignment of junction reads. We first examined the transcriptome for evidence of junction reads for the annotated HTR2A-AS1 gene. Only the annotated exon 2 was identified through an exon junction read in this dataset. However, our analysis of exonexon junction reads across canonical exon boundaries defined on the forward strand, agnostic to annotated HTR2A-AS1 exons, identified 15 additional exons. Four of these exons undergo apparent alternative splicing, resulting in a total of 22 discretely defined exons (Fig. 1b; Table 4).

Table 3 Putative Transcript Assembly for HTR2A

\begin{tabular}{ll}
\hline mRNA Exons Utilized & \# of Predicted TM Domains \\
\hline $1 \wedge 2 \wedge 3 \wedge 4$ & 7 \\
$0 \wedge 2 \wedge 3 \wedge 4$ & 7 \\
$1 \wedge 2 \operatorname{tr} \wedge 3 \wedge 4$ & 6 \\
$0 \wedge 3 \wedge 4$ & 5 \\
$1 \wedge 3 \wedge 4$ & 5 \\
$1 \wedge 2 \wedge 3 \wedge 3 b$ & 3 \\
$1 \wedge 2 \wedge 3 \wedge 3 a \wedge 4$ & 3 \\
$0 \wedge 3 \wedge 3 a \wedge 4$ & 3 \\
$0 \wedge 3 \wedge 3 b$ & No ORF \\
\hline
\end{tabular}


Table 4 HTR2A-AS1 Exons

\begin{tabular}{|c|c|c|c|c|c|}
\hline Exon & Size & Coordinates (hg19) ${ }^{a}$ & Predicted TSS ${ }^{\mathrm{b}}$ (hg19) & TSS Score ${ }^{b}$ & Notes \\
\hline 1 & $71^{c}$ & chr13:47370678-47370748 & chr13:47370715-47370719 & 0.8972 & \\
\hline 2 & $82^{c}$ & chr13:47426280-47426361 & chr13:47426295-47426299 & 0.5747 & As annotated in RefSeq \\
\hline 3 & 316 & chr13:47428074-47428389 & & & As annotated in RefSeq; No junction reads observed \\
\hline 3.1 & 144 & chr13:47428074-47428217 & & & \\
\hline 4 & $139^{c}$ & chr13:47429408-47429546 & chr13:47429250-47429253 & 0.2663 & \\
\hline 5 & $117^{c}$ & chr13:47429726-47429842 & chr13:47429797-47429804 & 0.5568 & \\
\hline 6 & 210 & chr13:47430225-47430434 & & & As annotated in RefSeq; No junction reads observed \\
\hline 7 & 133 & chr13:47466546-47466678 & & & Overlaps HTR2A exon 3 \\
\hline 7.1 & 194 & chr13:47466546-47466739 & & & Overlaps HTR2A exon 3 \\
\hline 8 & $101^{c}$ & chr13:47469779-47469879 & chr13:47469614-47469622 & 0.7864 & Overlaps HTR2A exon 2 \\
\hline 9 & $167^{c}$ & chr13:47471212-47471378 & chr13:47471046-47471066 & 0.9737 & Overlaps HTR2A exon 1ext \\
\hline 9.1 & 101 & chr13:47471282-47471382 & & & Overlaps HTR2A exon 1ext \\
\hline 10 & $101^{\mathrm{c}}$ & chr13:47472971-47473071 & chr13:47473021-47473029 & 0.7151 & \\
\hline 11 & $101^{c}$ & chr13:47474379-47474479 & chr13:47474236-47474243 & 0.7058 & \\
\hline 12 & $101^{c}$ & chr13:47476850-47476950 & chr13:47476912-47476920 & 0.7333 & \\
\hline 13 & 58 & chr13:47566052-47566109 & & & \\
\hline 14 & 46 & chr13:47595782-47595827 & & & \\
\hline 15 & 59 & chr13:47660942-47661000 & & & \\
\hline 16 & 29 & chr13:47748905-47748933 & & & \\
\hline 17 & 123 & chr13:47791699-47791821 & & & \\
\hline 17.1 & 119 & chr13:47791703-47791821 & & & \\
\hline 18 & 69 & chr13:47844316-47844384 & & & Contains canonical polyA signal \\
\hline
\end{tabular}

${ }^{\mathrm{a} C o o r d i n a t e s ~ r e f l e c t ~ h g 19 / G R C h 37 ~}$

${ }^{\mathrm{b}}$ Scores are predicted by Eponine (Down \& Hubbard, 2002) and includes 200 bp of upstream sequence. Only the highest score for each exon is reported

'No $5^{\prime}$ exon junction is defined, so size is estimated based on local read depth

The frequency of these exon junction reads varied widely (Additional file 6: Table S2), suggesting varying abundances of many different HTR2A-AS1 splice isoforms. Notably, multiple antisense exons overlapped sense-encoded HTR2A exons (Fig. 3a). Exons 1, 2, 4, 5, 8, 9, 9.1, 10, 11, and 12 did not have an exon junction read defining their 5 ' boundary, so we presume them to be transcription start sites. For these exons, we have used Eponine [32] to predict and score putative transcription start sites (Table 4). However, it is also possible that these exons represent internal exons, and we do not have adequate depth to capture all exonexon junctions, especially for exons with low expression. One canonical poly-A signal was observed in exon 18.

\section{Transcript assembly for HTR2A-AS1}

From the 22 different $H T R 2 A-A S 1$ exons we found to be expressed in the DLPFC, we inferred the transcript isoforms by examining the number of incoming junction reads spliced from upstream exons versus the outgoing junction reads going to downstream exons. Furthermore, we grouped junctions according to how often they were observed (number of samples, number of junction reads) into rare, low, medium, and high frequency groups, to aid our inference of transcript isoforms (Additional file 6: Table S2). Rare classification required only a single junction read in a single sample. Low, medium, and high classifications required a minimum of 2 reads in 2 samples, 25 reads in 25 samples, and 100 reads in 100 samples, respectively. Next, we exhaustively assembled 57 models of possible HTR2AAS1 RNA isoforms, which begin at transcription start sites, allow for only a single low coverage exon junction, and terminate at exons 17 or 18 (Additional file 7: Table S3 and HTR2A-AS1.FASTA). While we find it unlikely that 57 different transcripts are expressed from the HTR2A-AS1 locus, we used permissive criteria for assembly to next test for protein-coding potential of any possible antisense transcript. The transcript isoform models range in size from 248 to 626 nt. From all possible transcripts, the longest predicted open reading frame (ATG-to-stop) is only 31 amino acids long and is not supported as coding using the tcode program in EMBOSS [33]. Therefore, we find it likely that HTR2A-AS1 represents an lncRNA.

\section{Mouse Htr2a and Htr2a-AS1 alignments}

Using publically available RNA-Seq data generated from a variety of mouse tissues (Gene Expression Omnibus; 
GEO Accessions GSE36025, GSE52564, and GSE27243) [34-36], we analyzed mouse Htr2a and Htr2a-AS1 expression in a manner similar to the human brain tissues. The majority of reads mapped to the three annotated exons for Htr2a. However, we also found evidence for seven additional alternative sense-encoded exons (Additional file 8: Table S4). In the mouse, Htr2a exons 1 and 2 can be alternatively spliced to form low-abundance isoforms orthologous to human transcripts (Additional file 9: Figure S6). Of particular note, the highly-expressed archetype mouse isoform is orthologous to the human isoform that retains intron 1 . The mouse also expresses an orthologue to the human isoform in which exon 2 is spliced out. This is predicted to translate a protein that lacks the first two transmembrane domains, consistent with the human isoform lacking exon 2. We also observed infrequent utilization of the splice acceptor site equivalent to the human exon 2 truncation $\left(\mathrm{E} 2^{\mathrm{tr}}\right)$ that truncates the $\mathrm{N}$ terminus of $5-\mathrm{HT}_{2 \mathrm{~A}}$, resulting in a $6 \mathrm{TM}$ isoform. However, the splice donor site in mouse differs from that in humans, resulting in a predicted protein that includes all seven transmembrane domains, but lacks 71 of 75 amino acids constituting the $N$-terminus. Finally, much like human HTR2A, the mouse gene has a well-expressed extended 3' UTR, approximately $2.6 \mathrm{kB}$ longer than the current annotation, terminating at a canonical poly-A site (Additional file 10: Figure S7). The lack of a poly-A site (canonical or non-canonical) at the end of the current annotation and the contiguous high expression into the extended 3' UTR argues for revised annotation. As noted in Additional file 8: Table S4 and Additional file 9: Figure S6, we also see alternative splicing of the 3' UTR, which is predicted to shorten the protein by a single amino acid and change three terminal amino acids (...NEKVSCV vs. ...NEKMPF). This alternative isoform also excludes a large portion of the 3' UTR, the significance of which is unknown. Unlike human HTR2A, we do not see strong evidence for an extended 5' UTR in the mouse.

The mouse alignments suggest the presence of a lowabundance antisense transcript, extending approximately $96 \mathrm{kB}$ and comprised of up to 11 exons (Additional file 11: Table S5; Fig. 2). This antisense transcript also shares similarities to human HTR2A-AS1. In particular, the mouse expresses antisense isoforms that overlap sense-encoded exons of Htr2a and at similar positions as the human transcripts (Fig. 3b). The functional role for the antisense in regulating Htr2a function, if any, is not clear. However, the similarities in overlapping sense and antisense exons between mouse and human tissue lend support for a conserved regulatory function.

\section{Genetic influence of HTR2A and HTR2A-AS1 expression}

Guided by our newfound understanding of $H T R 2 A$ and HTR2A-AS1 gene structure, we tested the effects of the phenotype-associated SNPs rs6311 and rs6313 on various aspects of expression. To begin, we measured allelic RNA expression to identify influences of cis-acting genetic variants. Specifically, we tested whether rs6311 confers an allele-specific influence on the expression of the extended 5 ' UTR. Previously, the ancestral "G" allele was associated with increased expression relative to the variant " $\mathrm{A}$ " allele [21, 29]. Eighty-two of 109 DLPFC samples heterozygous rs6311had high-quality base calls over the rs6311 position, but low coverage precluded allelic analysis on individual samples. Instead we combined all of the reads at the rs6311 position across the 82 samples, observing significantly more reads of the " $G$ " allele relative to the " $\mathrm{A}$ " allele (187 vs 109 counts; binomial test $p=0.0004$ ), consistent with previous studies [21, 29]. We further tested whether rs6313 or rs6311 influenced expression of the highly expressed primary splice isoform in an allele-specific manner by measuring allelic expression at rs6313. Greater coverage at rs6313 allowed us to examine allelic expression directly at this SNP in individual samples, rather than relying on pooled counts across samples. If rs6311 or rs6313 were responsible for driving allele-specific expression of the primary transcript, our analysis should reveal imbalanced expression in most, if not all samples. However, only 9 of 109 samples heterozygous for rs6313 demonstrated significantly imbalanced expression ranging from 1.83 to 2.87-fold differences in expression across alleles (Table 5). The deviations from balanced expression suggest the presence of additional low frequency cis-acting regulatory variants, but argue strongly against a role for rs6311 or rs6313 in modulating expression of the highly-abundant HTR2A mRNA isoform lacking the extended 5' UTR.

In addition to direct allelic expression measures, we tested whether rs6311 or rs6313 are significantly associated with other HTR2A and HTR2A-AS1 expression measures, analogous to expression quantitative trait loci (eQTL). This included uniquely mapped reads covering all $H T R 2 A$ or HTR2A-AS1 exons, reads aligned to specific exons, and

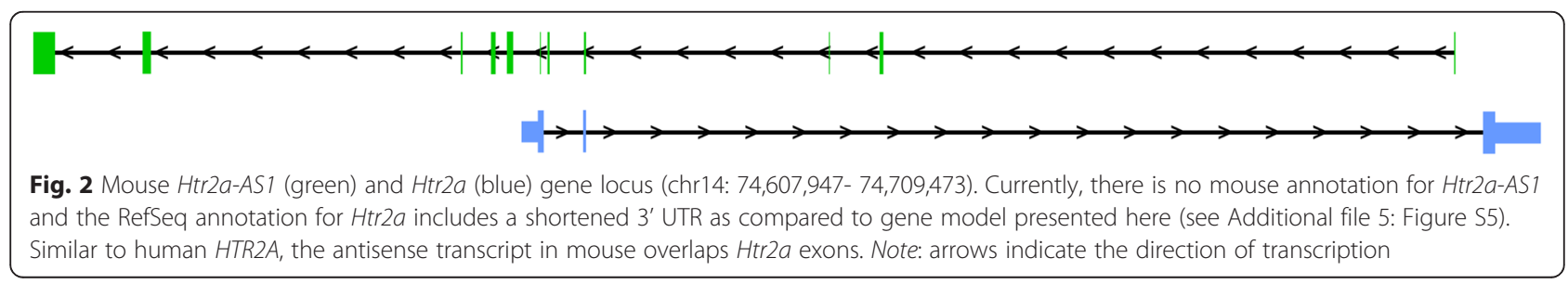



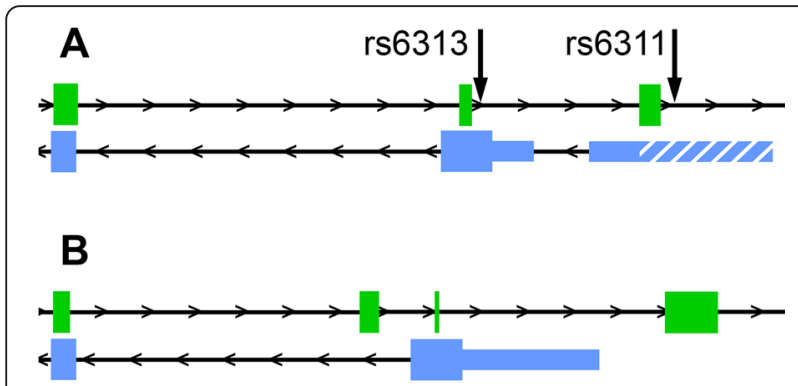

Fig. 3 Overlapping HTR2A (blue) and HTR2A-AS1 (green) exons in human (A) and mouse (B). a Antisense exons 7, 8, and 9 overlap human HTR2A exons 1, 2, and 3. Antisense exons 7 and 9 undergo alternative splicing, resulting in 5 unique exons that can overlap HTR2A transcripts. b Mouse antisense exons are situated similarly to the human antisense exons. Note: orientation of the mouse genes are reversed and mouse Htr2a exon 2 is aligned with human HTR2A exon 3 for comparison to the human genes. Arrows indicate the direction of transcription. Thin blue bars represent untranslated regions, with the hatched region of the human gene representing the extended $5^{\prime}$ UTR

junction reads for both $H T R 2 A$ and HTR2A-AS1 in the total DLPFC sample cohort. These analyses revealed no significant genotype-expression associations for HTR $2 A$ prior to Bonferroni correction. However, multiple aspects of HTR2A-AS1 expression were significantly associated with rs6311 and rs6313 genotypes, including total unique HTR2A-AS1 reads, HTR2A-AS1 exon 14 and 17.1 reads, and exon 14-17.1 junction reads (summarized in Table 6). Only the genotypic effect on exon 14 reads remained significant after strict Bonferroni correction. In all instances, increased expression of HTR2A-AS1 is associated with the variant alleles of rs6311 and rs6313 (A and T, respectively).

\section{Discussion}

Our analysis of RNA expression from the HTR2A gene locus provides us with valuable insight into the senseand antisense-encoded genes of this region, and delineates the use of known and novel exons. Of note, we see evidence for expression of up to 10 distinct senseencoded human HTR2A exons generated through the use of alternative transcription start sites, alternative splicing (intron retention, alternative donor site), and the infrequent use of novel splice donor/acceptor sites. On the antisense strand, we observed an abundance of novel HTR2A-AS1 exons. Including the previouslydescribed HTR2A-AS1 exons, our splice junction analysis provides evidence for up to 22 unique antisense exons, 5 of which overlap with sense-encoded HTR2A exons (Fig. 3a). Furthermore, we found evidence that the common SNPs rs6311 and rs6313 are associated with expression of the $H T R 2 A$ transcripts with the extended 5' UTR and HTR2A-AS1 exon 14. Finally, we demonstrated that the expression of Htr2a and Htr2a-AS1 in the mouse parallels expression in humans, suggesting that the mouse is suitable for studying specific sense and antisense isoforms.

\section{Consequences of HTR2A alternative splicing}

The genomics era, ushered in by massively-parallel DNA sequencing, has rapidly expanded our knowledge of gene architecture. At one time, nearly all GPCRs were considered to be without introns in their open reading frames (ORFs) [37]. While GPCRs are enriched for intronless ORFs relative to other protein classes, up to $42 \%$ of rhodopsin family GPCR genes have multiple exons that undergo alternative splicing [38]. Here, we found that HTR2A undergoes a variety of different splicing events, including utilization of alternative splice acceptor sites, exon skipping, rare exon usage, and intron retention, similar to previously described GPCR splicing patterns [39].

Inferred from splice junction reads, the majority of HTR2A transcripts encode for the full-length 7TM receptor (Table 3). However, some of the transcripts likely encode receptor proteins lacking the $\mathrm{N}$-terminus and varying stretches of transmembrane domains; particularly the isoforms with junctions shared between exon 0 and 3 and those utilizing exon $2^{\text {tr }}$. The previously described $6 \mathrm{TM}$ form resulting from usage of exon $2^{\text {tr }}$ [21] bears resemblance to other GPCRs reported to have truncated or "headless" forms: CALCR [40], CNR1 [41], CCKBR [42],

Table 5 Significant Allelic Expression Imbalance at rs6313

\begin{tabular}{lllllc}
\hline Sample & Diagnosis & G alleles & A alleles & Binom Dist $p$-val & Corrected $p$-val \\
\hline 1 & Scz & 92 & 36 & $3.94 \mathrm{E}-07$ & $4.30 \mathrm{E}-05$ \\
2 & Scz & 79 & 31 & $2.67 \mathrm{E}-06$ & $2.91 \mathrm{E}-04$ \\
3 & Control & 143 & 78 & $7.31 \mathrm{E}-06$ & $7.96 \mathrm{E}-04$ \\
4 & $\mathrm{Scz}$ & 95 & 48 & $5.25 \mathrm{E}-05$ & $5.72 \mathrm{E}-03$ \\
5 & $\mathrm{Scz}$ & 46 & 16 & $8.82 \mathrm{E}-05$ & $9.61 \mathrm{E}-03$ \\
6 & Scz & 67 & 30 & $1.09 \mathrm{E}-04$ & $1.19 \mathrm{E}-02$ \\
7 & Control & 69 & 32 & $1.48 \mathrm{E}-04$ & $1.61 \mathrm{E}-02$ \\
8 & Control & 57 & 26 & $4.39 \mathrm{E}-04$ & $4.78 \mathrm{E}-02$ \\
9 & Scz & 78 & 41 & $4.44 \mathrm{E}-04$ & $4.84 \mathrm{E}-02$ \\
\hline
\end{tabular}


Table 6 Associations Between rs6311 or rs6313 and RNA Expression

\begin{tabular}{|c|c|c|c|c|c|c|c|c|c|c|}
\hline \multirow[b]{2}{*}{ Gene } & \multicolumn{5}{|l|}{ rs6311 } & \multicolumn{5}{|l|}{ rs6313 } \\
\hline & $\mathrm{G} / \mathrm{G}(n)$ & $\mathrm{G} / \mathrm{A}(n)$ & $\mathrm{A} / \mathrm{A}(n)$ & $P$-Value ${ }^{a}$ & Adjusted P-Value & $C / C(n)$ & $C / T(n)$ & $\mathrm{T} / \mathrm{T}(n)$ & $P$-Value ${ }^{a}$ & Adjusted $P$-Value \\
\hline HTR2A-AS1 Unique Reads ${ }^{b}$ & $34.7(69)$ & $42.0(106)$ & $40.8(26)$ & 0.0070 & 0.4204 & $35.1(73)$ & $42.0(103)$ & $40.3(25)$ & 0.0153 & 0.9163 \\
\hline HTR2A Unique Reads ${ }^{c}$ & $4305.0(71)$ & $4525.7(111)$ & $3856.6(26)$ & 0.3026 & 1.0000 & $4262.2(75)$ & $4571.2(108)$ & $3934.5(25)$ & 0.3203 & 1.0000 \\
\hline HTR2A, AS1 Overlapping Reads ${ }^{\mathrm{d}}$ & $69.9(71)$ & 75.8 (111) & $62.0(26)$ & 0.3595 & 1.0000 & $69.2(75)$ & 75.8 (108) & $63.9(25)$ & 0.4112 & 1.0000 \\
\hline \multicolumn{11}{|l|}{ Exon } \\
\hline HTR2A-AS1 Exon 14 Reads ${ }^{\mathrm{e}}$ & $13.2(71)$ & $17.2(111)$ & $16.7(26)$ & 0.0004 & 0.0212 & $13.4(75)$ & $17.2(108)$ & $16.6(25)$ & 0.0010 & 0.0586 \\
\hline HTR2A-AS1 Exon 17.1 Reads ${ }^{f}$ & $16.4(69)$ & $21.3(106)$ & $19.6(26)$ & 0.0011 & 0.0687 & $16.6(73)$ & $21.3(103)$ & $19.6(25)$ & 0.0030 & 0.1771 \\
\hline \multicolumn{11}{|l|}{ Junction Reads } \\
\hline HTR2A-AS1 Exon 14 to 17.1 Reads $^{9}$ & $7.9(69)$ & 10.3 (108) & $9.8(27)$ & 0.0069 & 0.4169 & 8.1 (73) & $10.2(105)$ & $9.8(26)$ & 0.0142 & 0.8532 \\
\hline
\end{tabular}

Count values in the table are model predicted least square means. Differences in the subject numbers across outcomes reflect missing data in the selected variables for each model

${ }^{a}$ Calculated using a negative binomial generalized linear model with selection of covariates via Bayesian Information Criterion (BIC)

Covariates: ${ }^{a}$ alcohol use, RNA Integrity Number; ${ }^{c}$ gender, pH, RNA Integrity Number; ${ }^{d}$ gender, age, pH, RNA Integrity Number; ${ }^{~}$ RNA Integrity Number; ${ }^{f}$ alcohol use, post-mortem interval, RNA Integrity Number; galcohol use 
OPRL1 [43], OPRM1 [44], and SSTR5 [45]. While a headless form of $5-\mathrm{HT}_{2 \mathrm{~A}}$ would not necessarily disrupt the binding site for serotonin, it could abate membrane expression due to the deletion of $\mathrm{N}$-glycosylation sites [46]. A comparison across species at the nucleotide level reveals strong conservation beginning specifically at the splice acceptor site for exon $2^{\text {tr }}$ and a relative lack of amino acid conservation in the $\mathrm{N}$-terminus across species (Additional file 12: Figure S8). In our analysis of mouse $H t r 2 a$, we observed usage of the exon $2^{\text {tr }}$ splice site, which is predicted to result in a 7TM receptor that lacks almost the entire $N$ terminus. The $N$-glycosylation sites critical for $5-\mathrm{HT}_{2 \mathrm{~A}}$ membrane expression are conserved in mice, and are deleted by the use of the orthologous exon $2^{\text {tr }}$ splice site. Consequently, we speculate that membrane expression for the truncated receptor would be reduced in mice and humans.

It is not known whether the alternatively spliced mRNAs are translated into truncated $5-\mathrm{HT}_{2 \mathrm{~A}}$ protein isoforms in vivo, or subsequently capable of signaling from the cytoplasm. Assuming translation occurs, there are two observations arguing for the plausibility of intracellular $5-\mathrm{HT}_{2 \mathrm{~A}}$ signaling. First, serotonin that enters cells via the serotonin transporter is biologically active and involved in "serotonylation" of proteins [47]. Second, GPCRs in the cytoplasm, internalized via the endosome, can continue to signal through G protein-dependent and independent mechanisms [48]. Further studies characterizing the cell typespecific and developmental expression patterns of this truncated isoform could lend insights into the biological relevance of this, and perhaps other, headless GPCRs.

\section{HTR2A-AS1, a IncRNA}

Long non-coding RNAs are stringently defined by their length (>200 nt), lack of protein-coding potential, use of independent transcriptional units, presence of canonical splice site signals, and ability to undergo alternative splicing [49]. Some lncRNAs can have over 100 exons (SNURF$S N R P N)$ [50] and many are species-specific [51, 52]. With the additional exons and junctions we uncovered here, we reassessed classification of $H T R 2 A-A S 1$, firmly concluding that it meets criteria as an IncRNA.

The question of HTR2A-AS1 function remains. lncRNAs have diverse roles, but most characterized up to this point influence transcription and processing of RNAs from nearby genes, sometimes by direct RNA-RNA interactions, and other times through epigenetic modifications [53]. The fact that HTR2A-AS1 has exons directly overlapping with HTR2A transcripts (Fig. 3a), a feature which is also apparent in the mouse (Fig. 3b), argues strongly for the possibility of direct interaction through complementary nucleotide base-pairing, assuming the sense- and antisense-encoded transcripts are expressed at the same time and location. Direct RNA-RNA interactions could influence RNA processing, protein translation, or both. The relatively low abundance of the antisense transcripts relative to the sense isoforms and their apparent isoform diversity argues against a role in translation of the major HTR2A protein isoform, as these transcripts would have to exit the nucleus, find their respective targets, and impact the function of the translating ribosome.

Instead, we speculate that $H T R 2 A-A S 1$ is more likely to have roles in HTR2A mRNA expression or processing. The variable use of 5' exons in HTR2A-AS1 and Htr2a-AS1, coupled with their conserved overlap with alternativelyspliced HTR2A exons 1 and 2 (Fig. 3), could allow HTR2A$A S 1$ to participate in the alternative splicing of $H T R 2 A$ through direct RNA-RNA interactions. Additionally, the associations between the ancestral " $G$ " allele of rs6311, greater expression of HTR2A exon 1ext, and decreased expression of the seemingly constitutive HTR2A-AS1 exon 14 suggests a possible role in transcription start site usage or epigenetic modifications, perhaps through RNA-DNA interactions. In this case, we would presume that expression of the lncRNA is inversely associated with usage of the transcription start site that results in longer 5' UTR $H T R 2 A$ transcripts. It is also possible that $H T R 2 A-A S 1$ transcripts impact $H T R 2 A$ expression at multiple levels and in a species-specific manner. While molecular data showing an interaction of any kind between HTR2A-AS1 and HTR2A is lacking, our study provides critical information for testing these hypotheses, including evidence that an orthologous lncRNA is expressed in mice.

\section{HTR2A and HTR2A-AS1 genetics: rs6311 and rs6313}

HTR $2 A$ demonstrates many genotype-phentoype associations. A cursory analysis of the HuGE Navigator Genopedia shows 201 disease terms where HTR2A was investigated in 540 publications [54]. To put this into perspective, of the 12,488 genes listed in the HuGE Navigator, $H T R 2 A$ ranks $58^{\text {th }}$ with respect to the number of associated disease terms. Of the 540 HTR2A publications listed, 333 specifically mention rs6311 or rs6313 (or some variation of the nomenclature; e.g. $-1438 \mathrm{~A} / \mathrm{G}$, $\mathrm{T} 102 \mathrm{C}$, etc.) in their abstract or title, highlighting the interest in understanding disease associations conferred by these SNPs. While contradictory findings for any one phenotype-genotype association exist, overwhelming evidence implicates HTR $2 A$, and specifically rs6311 or rs6313, in a variety of phenotypes.

Because rs6311 and rs6313 are in near perfect LD, genetic association studies have the liberty of using either SNP to obtain similar results. However, each SNP must be considered separately when attempting to elucidate their biological functions and contributions to disease. To date, the most compelling evidence for modulating biological function supports a role for rs6311 in the expression of HTR2A mRNAs with the extended 5' UTR [21, 29], while no apparent role has been identified for rs6313. The allelic 
expression analysis of rs6311 in our current study supports the conclusion that rs6311 modulates expression of the extended 5' UTR. Aside from the infrequent allelic expression imbalances noted for rs6313, we find little evidence that expression of the highly-expressed primary HTR2A transcript not containing the extended 5' UTR is modulated by these common SNPs.

Regarding the transcription factors binding to the region of DNA harboring rs6311 to exert an allelic effect, the question remains open. An interesting candidate that unites genetic findings in schizophrenia is Early Growth Response 3 (EGR3). Polymorphisms in the promoter of the gene encoding EGR3 are implicated in schizophrenia [55]. Multiple studies have now demonstrated a link between $E G R 3,5-\mathrm{HT}_{2 \mathrm{~A}}$ receptor expression, and schizophrenialike behaviors in transgenic animals [56, 57]. The consensus binding site for EGR3 does align, although imperfectly, at the rs6311 region and the minor allele for rs6311 impacts this alignment (Additional file 13: Figure S9). Other transcription factors predicted to bind at the rs6311 locus in silico include the nuclear factor 1 family (NFIA/B/C), the Thing1/e47 heterodimer, and SMAD3 (Additional file 13: Figure S9). However, molecular evidence and additional bioinformatic studies are required to test such relationships, given the imprecision of transcription factor binding site tools in predicting relationships in vivo.

Changes in expression conferred by rs6311 or rs6313 could also result from other RNA processing events, such as alternative splicing. Considering the extent of alternative splicing on both the sense and antisense strands adjacent to rs6313, and the proximity of this SNP to alternative exons, we find it possible that this SNP could impact splicing. In silico analysis of splicing factors using a number of tools suggest this SNP can impact their binding (Additional file 14: Table S6). However, the aggregated results do not demonstrate a clear consensus for any single splicing factor being impacted by rs6313.

Future studies of HTR2A-AS1 expression should consider regulation by rs6311 or rs6313, as we found some evidence suggesting differential expression across genotype. However, the low read coverage across the entire HTR2A-AS1 transcript leads us to interpret these findings with caution. Both SNPs sit in intronic regions of HTR2A-AS1; rs6313 is 61 nucleotides downstream of exon 8 and rs6311 is 100 nucleotides downstream of exon 9. Both exons 8 and 9 share a relatively high number of splice junctions with the antisense exon 14 that exhibits genotype-expression associations. Our analyses are unable to isolate the individual contributions of either SNP due to high LD. Until stronger evidence delineates specific roles for each SNP, it remains equally plausible that either could modulate HTR2AAS1 expression.

\section{Conclusions}

We examined $H T R 2 A$ and $H T R 2 A-A S 1$ gene structure by mapping RNA-Seq junction reads from human prefrontal cortex, utilizing conventional definitions for exon structure independent of established gene annotations. This approach revealed two novel exons in HTR2A and showed the extent to which all exons are included in the mature mRNA transcripts. While the majority of transcripts consist of the previously-annotated exons that encode for the full-length 7TM protein, we also found evidence supporting the use of unannotated but previously-described exons that likely form truncated protein isoforms of $5-\mathrm{HT}_{2 \mathrm{~A}}$. In contrast, HTR2A-AS1 has numerous novel exons, some of which overlap with annotated HTR2A exons. The biological significance of HTR2A-AS1 transcripts is yet to be revealed, but the current findings enable studies to directly test if they regulate HTR2A expression. To that end, we conclude that the mouse could serve as a suitable model organism for studying whether sense-antisense interactions occur in Htr2a, as it also expresses many orthologous sense and antisense transcripts, and the overlapping features between the transcripts are spatially similar. Finally, we found evidence that the common genetic variant rs6311 regulates expression of $H T R 2 A$ transcripts containing the extended 5' UTR, consistent with previous studies. We also found associations between rs6311, rs6313, and expression of HTR2A-AS1. Guided by these findings, we can begin to examine variable transcript expression in the $H T R 2 A$ gene locus, especially focusing on diseases where it is implicated.

\section{Methods}

\section{Human tissues}

DLPFC specimens (Brodmann area 46/9) were dissected by a trained neuropathologist (T.M.H.) from brains collected post-mortem from the Office of the Chief Medical Examiner of the State of Maryland, with audiotaped informed consent from the legal next-of-kin, as approved by the Maryland Department of Health and Mental Hygiene's Institutional Review Board, as previously described $[58,59]$. Details of tissues acquisition, handling, processing, dissection, clinical characterization, diagnoses, and neuropathological examinations were also previously described [58]. Research on post-mortem samples was conducted in accordance with the U.S. Department of Health \& Human Services Code of Federal Regulations (Title 45, Part 46), in which post-mortem research is distinct from human subject research and not under the purview of a local IRB (http://www.hhs.gov/ohrp/ humansubjects/guidance/45cfr46.html\#46.102). Samples were de-identified and include 105 schizophrenia patients and 106 normal controls; 85 African Americans and 126 Caucasians (Demographics in Table 7). In accordance with 
Table 7 Human Tissue Demographics

\begin{tabular}{llll}
\hline & & Schizophrenia $(n=105)$ & Control $(n=106)$ \\
\hline Gender & Male & $74.3 \%(n=78)$ & $74.5 \%(n=79)$ \\
& Female & $25.7 \%(n=27)$ & $25.5 \%(n=27)$ \\
Race & Caucasian & $60.0 \%(n=63)$ & $59.4 \%(n=63)$ \\
& African-American & $40.0 \%(n=42)$ & $45.9 \pm 13.8$ years \\
Age at Death & & $45.6 \pm 13.8$ years & $82.1 \%(n=87)$ \\
Manner of Death & Natural & $59.0 \%(n=62)$ & $8.5 \%(n=9)$ \\
& Accidental & $13.3 \%(n=14)$ & $0.0 \%(n=0)$ \\
& Suicide & $26.7 \%(n=28)$ & $0.9 \%(n=1)$ \\
& Undetermined & $1.0 \%(n=1)$ & $0.9 \%(n=1)$ \\
No Autopsy & $0.0 \%(n=0)$ & $7.5 \%(n=8)$ \\
Smoking Status at Time of Death & Homicide & $0.0 \%(n=0)$ & $26.4 \%(n=28)$ \\
& Smoker & $70.5 \%(n=74)$ & $70.8 \%(n=75)$ \\
Brain pH & Non-smoker & $21.0 \%(n=22)$ & $2.8 \%(n=3)$ \\
Post-mortem interval & Not Determined & $8.6 \%(n=9)$ & $6.56 \pm 0.26$ \\
RNA Integrity Number & & $6.46 \pm 0.24$ & $29.4 \pm 14.1$ hours \\
\hline
\end{tabular}

known schizophrenia traits, smoking and suicide are noticeably higher in the affected cohort.

\section{Genotyping, illumina chips}

Genomic DNA was extracted from cerebellar tissues (Qiagen, Valencia CA, USA) and genotyped with HumanHap650Y_v3 or Human 1 M-Duo_v3 Illumina BeadChips (Illumina, San Diego, CA, USA) according to the manufacturer's instructions. Genotypes were called using the Illumina GenomeStudio v2010.1 software using the default settings and the chip-specific cluster files supplied by Illumina. For data analysis, SNPs had an overall missing rate $<0.02 \%$, CAUC and AA Hardy-Weinberg Equilibrium (HWE) p-values each $>=0.001$, and minor allele frequencies $(\mathrm{MAF})>=0.01$.

\section{RNA extraction and quality assessment}

Tissue from DLPFC was pulverized and stored at $-80{ }^{\circ} \mathrm{C}$. Total RNA was extracted from $100 \mathrm{mg}$ of tissue with TRIzol Reagent (Life Technologies, Grand Island, New York). The yield of total RNA was determined by spectrophotometry by measuring absorbance at $260 \mathrm{~nm}$. RNA quality was assessed with high-resolution capillary electrophoresis on an Agilent Bioanalyzer 2100 (Agilent Technologies, Palo Alto, California), yielding an RNA Integrity Number (RIN; scale $1-10$, with 1 being the lowest and 10 being the highest RNA quality).

\section{RNA-Seq library construction}

RNA-seq libraries were constructed using Illumina TruSeq RNA sample Prep Kit, following the manufacturer's protocol. The poly-A containing mRNA molecules were purified from $\sim 800 \mathrm{ng}$ DNAse treated total RNA. Following poly-A purification, the mRNA was fragmented into small pieces using divalent cations under elevated temperature $\left(94^{\circ}\right)$ for $2 \mathrm{~min}$. Under this condition, fragment lengths range from 130 to $290 \mathrm{bp}$ with a median length of $185 \mathrm{bp}$. Fragmented RNA was reverse transcribed into a first strand cDNA-RNA hybrid using random hexamers. Following, DNA Polymerase I and $\mathrm{RNaseH}$ were used to generate the complementary second strand cDNA. These double-stranded cDNA fragments then underwent end repair using T4 DNA polymerase, T4 PNK and Klenow DNA polymerase. Illumina PE barcoded adapters were ligated using T4 DNA Ligase following the addition of a single 'A' base using Klenow exo (3' to 5' exo minus). These products were then purified and PCR-enriched to create the final cDNA library for high through put DNA sequencing using a Highseq2000. The concentrations of RNA-seq libraries were measured by Qubit (Invitrogen, CA). The quality of each RNA-seq library was measured by LabChipGX (Caliper, MA) using HT DNA 1 K/12 K/ HiSens Labchip.

\section{Mouse tissues and sequencing}

All mouse data was obtained from public sources. Mouse tissues collection, RNA isolation, library preparation, and RNA-Seq were performed as described in their original publications [34-36]. Collected animal data conformed to local institutional review boards, as described in their original publications [34-36]. Raw BAM files from each of 
the three studies (Series Numbers GSE27243, GSE52564, and GSE36025) were downloaded from GEO (http:// www.ncbi.nlm.nih.gov/geo/) and aligned with GSNAP as described below to Mus musculus reference genome mm10.

\section{RNA sequence mapping}

Demultiplexing was performed with CASAVA v1.8.2 (http://support.illumina.com/sequencing/sequencing_software/casava.ilmn). Alignments were first performed with Tophat v2.0.4 [60] using the reference genome: Illumina UCSC hg19; and gene annotations: Ensembl GRCh37.67. An example command is: tophat -p 4 -r 160 -G Homo_sapiens.GRCh37.67.gtf -o Sample_out. Reads over the HTR2A region were extracted post processing with samtools v0.1.18 [61] with the following example command: samtools view -h Sample_RNA/accepted_hits.bam chr13: 47357513-47521169 > HTR2A_regions/Sample_RNA/ accepted_hits.sam. The extracted reads for each of the 211 samples were subsequently re-aligned to the human genome reference with GSNAP [62] which allows for gapped alignments, including intron-spanning alignments. Descriptive and statistical analyses were performed using this subsequent alignment.

\section{Alignment to the HTR2A locus}

On average, we generated 114 million reads per sample, $82 \%$ of which mapped to the reference genome (mapping statistics in Additional file 15: Table S1). HTR2A is on the reverse strand of chromosome 13 . Consequently, we defined canonical splice junctions with respect to transcription on the reverse strand and subsequently mapped junction reads across putative exons in order to define exon boundaries. In order for reads at novel junctions to be attributed to HTR2A, they must follow canonical splicing rules such that the nucleotides comprising the presumptive splice donor and acceptor sites are GU and AG, respectively. Adherence to this rule establishes the genomic DNA strand from which transcription occurred, eliminating ambiguity when assigning junction reads to $H T R 2 A$ versus the overlapping HTR2A-AS1. This resulted in an average of 7248 aligned reads per sample over the $H T R 2 A$ locus. Observational analysis, for the purpose of correcting for obvious bias in HTR2A mapping, revealed no overt differences across diagnosis or sex with respect to $H T R 2 A$ expression.

\section{RNA quantification}

Gene expression was quantified using the total number of reads for each sample that uniquely aligned to the reference. The read depth of each gene was computed based on the coordinates of mapped reads and newly annotated exons in the reference genome. Visualization of the mapping was carried out using publicly available software, primarily the Integrated Genomic Viewer (IGV) (http:// www.broadinstitute.org/software/igv/).

\section{Allelic expression imbalance}

Allele-specific expression was assessed for those samples that were heterozygous over rs6311 and rs6313. For measuring quality base counts, we used the following command, varying the region and sample: samtools mpileup $-\mathrm{uD}-\mathrm{r}$ chr13:47357513-47521169 -f ref_genome.fa R3924_".bam | bcftools view -cg - I grep 47469940. Read counts from the DP4 field was used in a binomial statistical test with a $5 \%$ multiple test corrected significance cut-off.

\section{In silico splicing and transcription factor binding analysis}

Sequences $100 \mathrm{bp}$ upstream and downstream of exons was downloaded from UCSC Genome Browser and submitted to the Human Splice Finder v2.4.1 in silico webserver (http://www.umd.be/HSF/), which scores splicing characteristics (splice donor site, acceptor site, branch point) [30]. For the branch point analysis, the highest scoring motif between positions -50 and -17 relative to the exon start site was considered for analysis. Positions -40 to -5 were analyzed for polypyrimidine frequency, expressed as a percentage.

Splicing characteristics were also scored using the Alternative Splice Site Predictor [31]. For this analysis, sequence for the entire HTR2A region was downloaded via the UCSC Genome Browser and submitted to the webserver (http://wangcomputing.com/assp/index.html) for scoring. We compared the average of scores obtained for annotated RefSeq exons vs. newly-discovered exons vs. all other predicted splice donor/acceptor sites using independent sample t-tests. In-depth analysis of splicing events for rs6313 alleles used multiple in silico tools for predicting putative splicing-related proteins [63-67].

Transcription factor binding for rs6311 alleles was performed using MatInspector (http://www.genomatix.de/ matinspector) [68].

\section{Statistical methods}

A natural choice to model read counts is the Poisson distribution. However, it has been shown that Poisson distribution does not capture biological variation in read counts [69-71]. To account for the extra variation found in biological replicates, the Poisson distribution can be extended through the negative binomial distribution, which has been used extensively to model RNA-Seq data [72, 73]. Thus, a generalized linear model (GLM) with logit link function using the negative binomial distribution was used to analyze read counts. The genotypic model was $\log$ (UniquelyMappedReads $*$ ExpressionRate) $=$ Covariates + SNP. The best set of covariates was chosen using the model that minimized the Bayesian Information Criterion. Covariates considered were age, race, gender, diagnosis, alcohol use, 
smoking status, PMI, brain $\mathrm{pH}, \mathrm{RIN}$, and suicide. The brain $\mathrm{pH}$ and RIN were the main covariates explaining significant amounts of read count variability and were included in most models. Multiplicity correction was performed using a Bonferroni adjustment for the number of endpoints tested times the effective number of independent SNPs tested [74], to control type I error at an experiment-wise alpha level of 0.2 .

\section{Availability of supporting data}

Human DLPFC RNA-Seq and genotyping data will be available for download in early 2016 according to the data sharing policy described Schubert et al. (2015) [75]. Mouse data used in this study can be downloaded from GEO at http://www.ncbi.nlm.nih.gov/geo/query/acc.cgi? acc=GSE27243 [34], http://www.ncbi.nlm.nih.gov/geo/ query/acc.cgi?acc=GSE52564 [35], and http://www.ncbi. nlm.nih.gov/geo/query/acc.cgi?acc=GSE36025 [36]. Mapping software used in the current study includes CASAVA 1.8.2 (http://support.illumina.com/sequencing/ sequencing_software/casava.ilmn), TopHat 2.0.4 (https:// ccb.jhu.edu/software/tophat/index.shtml) [60], samtools v0.1.18 (http://samtools.sourceforge.net/) [61], and GSNAP (http://research-pub.gene.com/gmap/) [62]. Aligned .BAM files were viewed using IGV (https://www.broadinstitute.org/igv/). Splicing analyses were performed using Human Splice Finder v2.4.1 (http://www.umd.be/HSF/) [30], Alternative Splice Site Predictor (http://wangcomputing.com/ assp/index.html) [31], SpliceAid2 (http://www.introni.it/ splicing.html) [63], Rescue-ESE (http://genes.mit.edu/burgelab/rescue-ese/) [64], FAS-ESS (http://genes.mit.edu/fasess/) [65], SFmap (http://sfmap.technion.ac.il) [66], ACEScan (http://genes.mit.edu/acescan2/) [67]. Transcription factor analysis was performed using MatInspector (http:// www.genomatix.de/matinspector) [68].

\section{Additional files}

\section{Additional file 1: Figure S1. Revised gene model for human HTR2A. (PDF $112 \mathrm{~kb}$ )}

Additional file 2: Figure S2. Predicted splice donor, splice acceptor, branch site, and polypyrimidine tract scores for annotated vs. novel HTR2A exons. (PDF $144 \mathrm{~kb}$ )

Additional file 3: Figure S3. Reads mapped at the $5^{\prime} U T R$ of human HTR2A and predicted transcription start sites, visualized using IGV. (PDF $102 \mathrm{~kb}$ )

Additional file 4: Figure S4. Reads mapped at the $3^{\prime} U T R$ of human HTR2A and predicted polyadenylation signals, visualized using IGV. (PDF $108 \mathrm{~kb}$ )

Additional file 5: Figure S5. Reads mapped at exon $3 \mathrm{~b}$ of human HTR2A and predicted polyadenylation signals, visualized using IGV. (PDF 105 kb)

Additional file 6: Table S2. Splice junctions and counts for human HTR2A-AS1. (XLSX $12 \mathrm{~kb}$ )

Additional file 7: Table S3. Predicted HTR2A-AS1 transcripts and open reading frames. (XLSX $13 \mathrm{~kb}$ )

Additional file 8: Table S4. Mouse Htr2a exons and coordinates. (XLSX $12 \mathrm{~kb})$
Additional file 9: Figure S6. Revised gene model for mouse Htr2a. (PDF $93 \mathrm{~kb}$ )

Additional file 10: Figure S7. Reads mapped the $3^{\prime} U T R$ of mouse Htr2a and predicted polyadenylation signals, visualized using IGV. (PDF 101 kb)

Additional file 11: Table S5. Mouse Htr2a-AS1 exons and coordinates. (XLSX $12 \mathrm{~kb}$ )

Additional file 12: Figure S8. Predicted conservation of $5-\mathrm{HT}_{2 \mathrm{~A}}$ protein across 9 species. (PDF $165 \mathrm{~kb}$ )

Additional file 13: Figure S9. Putative transcription factor binding sites at the rs6311 locus in human HTR2A. (PDF 106 kb)

Additional file 14: Table S6. Predicted splicing proteins bound to human HTR2A rs6313 alleles. (XLSX 11 kb)

Additional file 15: Table S1. Mapping statistics for human DLPFC RNA-Seq samples (XLSX $22 \mathrm{~kb}$ )

\section{Competing interests}

CLR, RMS, JC, LM, DCA and LKN are employees and stockholders of Eli Lilly and Company. YG, JHS, TH and RES have no competing interests. DRW serves on the Advisory Council of Sunovion Pharma. This study was funded by Eli Lilly and Company.

\section{Authors' contributions}

CLR and RMS designed experiments, analyzed data, and wrote the manuscript. JC wrote software used for junction mapping and analysis and consulted on interpretation. DCA performed statistical analyses and edited the manuscript. LM designed experiments, obtained data, and guided statistical analyses. YG designed experiments and developed the RNA-Seq analysis pipeline. JHS executed experiments and performed RNA-Seq quality control. TH designed experiments, collected data, and edited the manuscript. RES performed genotype data quality control, consulted on interpretation, and edited the manuscript. DRW designed experiment, established RNA-Seq benchmarks, and reviewed data. LKN designed experiments, obtained data, guided statistical analysis, consulted on interpretation, and edited the manuscript. All authors have read and approved the final version of the manuscript.

\section{Acknowledgements}

This study was funded by Eli Lilly and Company.

\section{Author details}

${ }^{1}$ Tailored Therapeutics, Lilly Corporate Center, Eli Lilly and Company,

Indianapolis, IN, . . 'Lieber Institute for Brain Development, Baltimore, MD,

${ }^{3}$ Departments of Neurology, Psychiatry and Behavioral Sciences, John Hopkins University School of Medicine, Baltimore, MD, . . ${ }^{4}$ Departments of Psychiatry, Neurology, Neuroscience, and the Institute of Genetic Medicine, Johns Hopkins University School of Medicine, Baltimore, MD,

Received: 9 September 2015 Accepted: 22 December 2015 Published online: 06 January 2016

\section{References}

1. Celada P, Puig MV, Artigas F. Serotonin modulation of cortical neurons and networks. Front Integr Neurosci. 2013;7:25.

2. De Clerck F, Xhonneux B, Leysen J, Janssen PA. Evidence for functional 5-HT2 receptor sites on human blood platelets. Biochem Pharmacol. 1984;33(17): 2807-11.

3. Cohen ML, Fuller RW, Wiley KS. Evidence for 5-HT2 receptors mediating contraction in vascular smooth muscle. J Pharmacol Exp Ther. 1981;218(2): 421-5.

4. McLennan PL, Taylor DA. Antagonism by ketanserin of 5-HT-induced vasoconstriction unmasks a 5-HT-induced vasodilation. Eur J Pharmacol. 1984;104(3-4):313-8.

5. Nau Jr F, Yu B, Martin D, Nichols CD. Serotonin 5-HT2A receptor activation blocks TNF-a mediated inflammation in vivo. PLoS One. 2013;8(10), e75426.

6. Van de Kar LD, Javed A, Zhang Y, Serres F, Raap DK, Gray TS. 5-HT2A receptors stimulate $\mathrm{ACTH}$, corticosterone, oxytocin, renin, and prolactin release and activate hypothalamic CRF and oxytocin-expressing cells. J Neurosci. 2001;21(10):3572-9. 
7. Burnet PW, Eastwood SL, Lacey K, Harrison PJ. The distribution of 5-HT1A and 5-HT2A receptor mRNA in human brain. Brain Res. 1995;676(1):157-68.

8. Jakab RL, Goldman-Rakic PS. 5-Hydroxytryptamine2A serotonin receptors in the primate cerebral cortex: possible site of action of hallucinogenic and antipsychotic drugs in pyramidal cell apical dendrites. Proc Natl Acad Sci U S A. 1998;95(2):735-40

9. DisGeNET: a discovery platform for the dynamical exploration of human diseases and their genes. HTR2A-disease association data were retrieved from the DisGeNET Database, GRIB/IMIM/UPF Integrative Biomedical Informatics Group, Barcelona, on 10/14/2014 [http://www.disgenet.org/].

10. Cummings J, Isaacson S, Mills $\mathrm{R}$, Williams $\mathrm{H}$, Chi-Burris $\mathrm{K}$, Corbett $\mathrm{A}$, et al. Pimavanserin for patients with Parkinson's disease psychosis: a randomised, placebo-controlled phase 3 trial. Lancet. 2014;383(9916):533-40.

11. Gasque G, Conway S, Huang J, Rao Y, Vosshall LB. Small molecule drug screening in Drosophila identifies the 5HT2A receptor as a feeding modulation target. Sci Rep. 2013;3:srep02120.

12. Moldofsky H, Harris HW, Archambault WT, Kwong T, Lederman S. Effects of bedtime very low dose cyclobenzaprine on symptoms and sleep physiology in patients with fibromyalgia syndrome: a double-blind randomized placebo-controlled study. J Rheumatol. 2011;38(12):2653-63.

13. ClinicalTrials.Gov: A Study to Evaluate the Effects of RCN3028 on Moderate to Severe Vasomotor Symptoms in Women (RDC) [http://clinicaltrials.gov/ show/NCT02100540].

14. Lianoglou S, Garg V, Yang JL, Leslie CS, Mayr C. Ubiquitously transcribed genes use alternative polyadenylation to achieve tissue-specific expression. Genes Dev. 2013;27(21):2380-96.

15. Wang ET, Sandberg R, Luo S, Khrebtukova I, Zhang L, Mayr C, et al. Alternative isoform regulation in human tissue transcriptomes. Nature. 2008; 456(7221):470-6.

16. Barrie ES, Smith RM, Sanford JC, Sadee W. mRNA transcript diversity creates new opportunities for pharmacological intervention. Mol Pharmacol. 2012; 81(5):620-30.

17. Saltzman AG, Morse B, Whitman MM, Ivanshchenko $Y$, Jaye M, Felder S. Cloning of the human serotonin 5-HT2 and 5-HT1C receptor subtypes. Biochem Biophys Res Commun. 1991;181(3):1469-78.

18. Chen K, Yang W, Grimsby J, Shih JC. The human 5-HT2 receptor is encoded by a multiple intron-exon gene. Brain Res Mol Brain Res. 1992;14(1-2):20-6.

19. Julius D, Huang KN, Livelli TJ, Axel R, Jessell TM. The 5hT2 receptor defines a family of structurally distinct but functionally conserved serotonin receptors. Proc Natl Acad Sci U S A. 1990;87(3):928-32.

20. Guest PC, Salim K, Skynner HA, George SE, Bresnick JN, McAllister G. Identification and characterization of a truncated variant of the 5-hydroxytryptamine(2A) receptor produced by alternative splicing. Brain Res. 2000;876(1-2):238-44.

21. Smith RM, Papp AC, Webb A, Ruble CL, Munsie LM, Nisenbaum LK, et al. Multiple regulatory variants modulate expression of 5-hydroxytryptamine 2A receptors in human cortex. Biol Psychiatry. 2013;73(6):546-54.

22. Liu W, Downing AC, Munsie LM, Chen P, Reed MR, Ruble $C L$, et al. Pharmacogenetic analysis of the mGlu2/3 agonist LY2140023 monohydrate in the treatment of schizophrenia. Pharmacogenomics J. 2012;12(3):246-54.

23. GTEx Consortium. The Genotype-Tissue Expression (GTEx) project. Nat Genet. 2013:45(6):580-5.

24. Saiz PA, Garcia-Portilla MP, Paredes B, Arango C, Morales B, Alvarez V, et al. Association between the A-1438G polymorphisms of the serotonin 2A receptor gene and nonimpulsive suice attempts. Psychiatr Genet. 2008;18(5):213-8.

25. Abdolmaleky HM, Yaqubi S, Papageorgis P, Lambert AW, Ozturk S, Sivaraman V, et al. Epigenetic dysregulation of HTR2A in the brain of patients with schizophrenia and bipolar disorder. Schizophr Res. 2011; 129(2-3):183-90.

26. Turecki $G$, Brière $R$, Dewar $K$, Antonetti $T$, Lesage $A D$, Séguin $M$, et al. Prediction of level of serotonin $2 A$ receptor binding by serotonin receptor 2A genetic variation in postmortem brain samples from subjects who did or did not commit suicide. Am J Psychiatry. 1999;156(9):1456-8.

27. Bray NJ, Buckland PR, Hall H, Owen MJ, O'Donovan MC. The serotonin-2A receptor gene locus does not contain common polymorphism affecting mRNA levels in adult brain. Mol Psychiatry. 2004;9(1):109-14.

28. Fukuda $Y$, Koga $M$, Arai M, Noguchi E, Ohtsuki T, Horiuchi $Y$, et al. Monoallelic and unequal allelic expression of the HTR2A gene in human brain and peripheral lymphocytes. Biol Psychiatry. 2006;60(12):1331-5.

29. Smith RM, Banks W, Hansen E, Sadee W, Herman GE. Family-based clinical associations and functional characterization of the serotonin 2A receptor gene (HTR2A) in autism spectrum disorder. Autism Res. 2014;7(4):459-67.
30. Desmet FO, Hamroun D, Lalande M, Collod-Béroud G, Claustres M, Béroud C. Human Splicing Finder: an online bioinformatics tool to predict splicing signals. Nucleic Acids Res. 2009;37(9), e67.

31. Wang M, Marín A. Characterization and prediction of alternative splice sites. Gene. 2006;366(2):219-27.

32. Down TA, Hubbard TJ. Computational detection and location of transcription start sites in mammalian genomic DNA. Genome Res. 2002;12(3):458-61.

33. Rice P, Longden I, Bleasby A. EMBOSS: the European Molecular Biology Open Software Suite. Trends Genet. 2000;16(6):276-7.

34. Belgard TG, Marques AC, Oliver PL, Abaan HO, Sirey TM, Hoerder-Suabedissen A, et al. A transcriptomic atlas of mouse neocortical layers. Neuron. 2011;71(4): 605-16.

35. Lin S, Lin Y, Nery JR, Urich MA, Breschi A, Davis CA, et al. Comparison of the transcriptional landscapes between human and mouse tissues. Proc Natl Acad Sci U S A. 2014;111(48):17224-9.

36. Zhang Y, Chen K, Sloan SA, Bennett ML, Scholze AR, O'Keeffe S, et al. An RNA-sequencing transcriptome and splicing database of glia, neurons, and vascular cells of the cerebral cortex. J Neurosci. 2014;34(36):11929-47.

37. Gentles AJ, Karlin S. Why are human G-protein-coupled receptors predominantly intronless? Trends Genet. 1999;15(2):47-9.

38. Markovic D, Challiss RA. Alternative splicing of $G$ protein-coupled receptors: physiology and pathophysiology. Cell Mol Life Sci. 2009;66(20): 3337-52.

39. Einstein R, Jordan H, Zhou W, Brenner M, Moses EG, Liggett SB. Alternative splicing of the $G$ protein-coupled receptor superfamily in human airway smooth muscle diversifies the complement of receptors. Proc Natl Acad Sci U S A. 2008;105(13):5230-5.

40. Nag K, Sultana N, Kato A, Hirose S. Headless splice variant acting as dominant negative calcitonin receptor. Biochem Biophys Res Commun. 2007:362(4):1037-43.

41. Shire D, Carillon C, Kaghad M, Calandra B, Rinaldi-Carmona M, Le Fur G, et al. An amino-terminal variant of the central cannabinoid receptor resulting from alternative splicing. J Biol Chem. 1995;270(8):3726-31.

42. Miyake A. A truncated isoform of human CCK-B/gastrin receptor generated by alternative usage of a novel exon. Biochem Biophys Res Commun. 1995; 208(1):230-7.

43. Pan $Y X, X u$ J, Wan BL, Zuckerman A, Pasternak GW. Identification and differential regional expression of KOR-3/ORL-1 gene splice variants in mouse brain. FEBS Lett. 1998;435(1):65-8.

44. Majumdar S, Grinnell S, Le Rouzic V, Burgman M, Polikar L, Ansonoff M, et al Truncated G protein-coupled mu opioid receptor MOR-1 splice variants are targets for highly potent opioid analgesics lacking side effects. Proc Natl Acad Sci U S A. 2011;108(49):19778-83.

45. Cordoba-Chacon J, Gahete MD, Duran-Prado M, Luque RM, Castano JP. Truncated somatostatin receptors as new players in somatostatin-cortistatin pathophysiology. Ann N Y Acad Sci. 2011;1220:6-15.

46. Maginnis MS, Haley SA, Gee GV, Atwood WJ. Role of N-linked glycosylation of the 5-HT2A receptor in JC virus infection. J Virol. 2010;84(19):9677-84.

47. Walther DJ, Peter JU, Winter S, Höltje M, Paulmann N, Grohmann M, et al. Serotonylation of small GTPases is a signal transduction pathway that triggers platelet alpha-granule release. Cell. 2003;115(7):851-62.

48. Calebiro D, Nikolaev VO, Persani L, Lohse MJ. Signaling by internalized Gprotein-coupled receptors. Trends Pharmacol Sci. 2010;31(5):221-8.

49. Derrien $T$, Johnson R, Bussotti G, Tanzer A, Djebali S, Tilgner $H$, et al. The GENCODE $\mathrm{V} 7$ catalog of human long noncoding RNAs: analysis of their gene structure, evolution, and expression. Genome Res. 2012;22(9):1775-89.

50. Runte M, Hüttenhofer A, Gross S, Kiefmann M, Horsthemke B, Buiting K. The IC-SNURF-SNRPN transcript serves as a host for multiple small nucleolar RNA species and as an antisense RNA for UBE3A. Hum Mol Genet. 2001;10(23): 2687-700

51. Paralkar VR, Mishra T, Luan J, Yao Y, Kossenkov AV, Anderson SM, et al. Lineage and species-specific long noncoding RNAs during erythro-megakaryocytic development. Blood. 2014;123(12):1927-37.

52. Necsulea A, Soumillon M, Warnefors M, Liechti A, Daish T, Zeller U, et al. The evolution of IncRNA repertoires and expression patterns in tetrapods. Nature. 2014;505(7485):635-40.

53. Cech TR, Steitz JA. The noncoding RNA revolution-trashing old rules to forge new ones. Cell. 2014;157(1):77-94.

54. Yu W, Clyne M, Khoury MJ, Gwinn M. Phenopedia and Genopedia: diseasecentered and gene-centered views of the evolving knowledge of human genetic associations. Bioinformatics. 2010;26(1):145-6. 
55. Yamada K, Gerber DJ, Iwayama Y, Ohnishi T, Ohba H, Toyota T, et al Genetic analysis of the calcineurin pathway identifies members of the EGR gene family, specifically EGR3, as potential susceptibility candidates in schizophrenia. Proc Natl Acad Sci U S A. 2007;104(8):2815-20.

56. Maple AM, Zhao X, Elizalde DI, McBride AK, Gallitano AL. Htr2a Expression Responds Rapidly to Environmental Stimuli in an Egr3-Dependent Manner. ACS Chem Neurosci. 2015;6(7):1137-42

57. Williams AA, Ingram WM, Levine S, Resnik J, Kamel CM, Lish JR, et al. Reduced levels of serotonin $2 \mathrm{~A}$ receptors underlie resistance of Egr3-deficient mice to locomotor suppression by clozapine. Neuropsychopharmacology. 2012;37(10): 2285-98.

58. Lipska BK, Deep-Soboslay A, Weickert CS, Hyde TM, Martin CE, Herman MM, et al. Critical factors in gene expression in post-mortem human brain: Focus on studies in schizophrenia. Biol Psychiatry. 2006;60(6):650-8.

59. Jaffe AE, Shin J, Collado-Torres L, Leek JT, Tao R, Li C, et al. Developmental regulation of human cortex transcription and its clinical relevance at single base resolution. Nat Neurosci. 2015;18(1):154-61.

60. Kim D, Pertea G, Trapnell C, Pimentel H, Kelley R, Salzberg SL. TopHat2: accurate alignment of transcriptomes in the presence of insertions, deletions and gene fusions. Genome Biol. 2013;14(4):R36.

61. Li H, Handsaker B, Wysoker A, Fennell T, Ruan J, Homer N, et al. The Sequence Alignment/Map format and SAMtools. Bioinformatics. 2009;25(16):2078-9.

62. Wu TD, Nacu S. Fast and SNP-tolerant detection of complex variants and splicing in short reads. Bioinformatics. 2010;26(7):873-81.

63. Piva F, Giulietti M, Burini AB, Principato G. SpliceAid 2: a database of human splicing factors expression data and RNA target motifs. Hum Mutat. 2012; 33(1):81-5.

64. Fairbrother WG, Yeh RF, Sharp PA, Burge CB. Predictive identification of exonic splicing enhancers in human genes. Science. 2002;297(5583):1007-13.

65. Wang Z, Rolish ME, Yeo G, Tung V, Mawson M, Burge CB. Systematic identification and analysis of exonic splicing silencers. Cell. 2004;119(6):831-45.

66. Paz I, Akerman M, Dror I, Kosti I, Mandel-Gutfreund Y. SFmap: a web server for motif analysis and prediction of splicing factor binding sites. Nucleic Acids Res. 2010;38:W281-5.

67. Yeo GW, Van Nostrand E, Holste D, Poggio T, Burge CB. Identification and analysis of alternative splicing events conserved in human and mouse. Proc Natl Acad Sci U S A. 2005;102(8):2850-5

68. Cartharius K, Frech K, Grote K, Klocke B, Haltmeier M, Klingenhoff A, et al. MatInspector and beyond: promoter analysis based on transcription factor binding sites. Bioinformatics. 2005;21(13):2933-42.

69. Nagalakshmi U, Wang Z, Waern K, Shou C, Raha D, Gerstein M, et al. The transcriptional landscape of the yeast genome defined by RNA sequencing. Science. 2008;320(5881):1344-9.

70. Robinson MD, Smyth GK. Moderated statistical tests for assessing differences in tag abundance. Bioinformatics. 2007;23(21):2881-7.

71. Bullard JH, Purdom E, Hansen KD, Dudoit S. Evaluation of statistical methods for normalization and differential expression in mRNA-Seq experiments. BMC Bioinformatics. 2010;11:94

72. Anders S, Huber W. Differential expression analysis for sequence count data. Genome Biol. 2010;11(10):R106.

73. Robinson MD, McCarthy DJ, Smyth GK. edgeR: a Bioconductor package for differential expression analysis of digital gene expression data. Bioinformatics. 2010;26(1):139-40.

74. Gao X, Starmer J, Martin ER. A multiple testing correction method for genetic association studies using correlated single nucleotide polymorphisms. Genet Epidemiol. 2008;32(4):361-9.

75. Schubert CR, O'Donnell P, Quan J, Wendland JR, Xi HS, Winslow AR, et al. BrainSeq: neurogenomics to drive novel target discovery for neuropsychiatric disorders. Neuron. 2015;88(6):1078-83.

\section{Submit your next manuscript to BioMed Central and we will help you at every step:}

- We accept pre-submission inquiries

- Our selector tool helps you to find the most relevant journal

- We provide round the clock customer support

- Convenient online submission

- Thorough peer review

- Inclusion in PubMed and all major indexing services

- Maximum visibility for your research

Submit your manuscript at www.biomedcentral.com/submit
Biomed Central 